Original Research Article

\title{
Anti-microbial sensitivity and resistance of organisms in blood-culture samples from prolonged fever cases: evidence from a tertiary care hospital in West Bengal, India
}

\author{
Somanjana Ghosh ${ }^{1}$, Arista Lahiri ${ }^{2}$, Siddharatha Bera ${ }^{3}$, Soumyajyoti Bandyopadhyay ${ }^{4 *}$
}

${ }^{1}$ Department of Microbiology, Midnapore Medical College \& Hospital, Midnapore, West Bengal, India

${ }^{2}$ Department of Community Medicine, Medical College \& Hospital, Kolkata, West Bengal, India

${ }^{3}$ Student, College of Medicine \& Sagore Dutta Hospital, Kamarhati, West Bengal, India ${ }^{4}$ Department of Forensic Medicine \& Toxicology, College of Medicine \& JNM Hospital, Kalyani, West Bengal, India

Received: 22 August 2018 Accepted: 27 September 2018

*Correspondence to:

Dr. Soumyajyoti

Bandyopadhyay,

Email: dr.soumyajyoti@ gmail.com

Copyright: (C) the author(s), publisher and licensee Medip Academy. This is an openaccess article distributed under the terms of the Creative Commons Attribution NonCommercial License, which permits unrestricted noncommercial use, distribution, and reproduction in any medium, provided the original work is properly cited.

\begin{abstract}
Background: Currently there is a rise in resistance to anti-microbials which is a matter of concern in treatment of systemic infections. Blood culture is considered "gold standard" in diagnosis of suspected systemic infection. The susceptibility to antibiotics thereafter determine the future course of treatment. The current study aims to find out the sensitivity and resistance pattern of the blood culture isolates.
\end{abstract}

Methods: A cross-sectional study was performed on the blood culture samples sent within 24hours of admission of the adult patients reporting fever for 7 days or more with no history of consumption of any antibiotics within last month. Total 134 blood samples were analysed. The proportion of sensitivity and resistance to anti-bacterial agents was calculated among those samples which showed growth in the culture. Background information of the patients in terms of age, sex and religion were also noted.

Results: Mean age of the patients was $39.33( \pm 12.19)$ years. Overall $47.76 \%$ were female patients and remaining were male. Among the Hindu patients majority were male while among Muslims majority were female. Of the total number of blood cultures examined $46.27 \%$ showed growth of bacteria. Staphylococcus aureus was the most frequently found bacteria isolated in cultures, followed by coagulase negative Staphylococcus and Pseudomonas. Of the frequently used antibiotics, higher sensitivity was seen with vancomycin, amikacin, netilmycin, imipenem, gentamicin. High resistance was observed in use of antibiotics like cefixime, amoxicillin-clavulanic acid and azithromycin.

Conclusions: High level of resistance to several commonly used advanced antibiotics warrant judicial and evidence-based use of these drugs.

Keywords: Antibiotic, Anti-microbial, Blood culture, Culture and sensitivity, Resistance, Sensitivity, Susceptibility

\section{INTRODUCTION}

Pyrexia or fever in general has been a talismanic contributor to morbidity and mortality in the human civilization since historic times. As understood conceptually anything increasing the burden of morbidity and mortality, will also incur financial loss to the society with fever, infection, sepsis being no exception. ${ }^{1-3}$ Though 
fever can be manifestation of any form of systemic inflammation including but not limited to infections, still in clinical practice a well-founded presumption in fever of long duration is to identify the source of infection. Thus, establishing the source and offending organisms, which will help in initiating the targeted therapy.

In the pre-antibiotic era the burden of pyrexia due to infection was very high. ${ }^{4}$ Infections usually led to death or severe morbidity. However, antibiotics thereafter were a cardinal stepping stone in evolution of the human civilization. After the advent of antibiotics, the burden of infection, fever, sepsis decreased significantly. ${ }^{1,4}$ But with time, indiscriminate and unscientific and often marketingdriven use of antibiotics have led to development of resistance to certain antibiotics by the several of the pathogens. The pattern of resistance has further moulded into a highly resistant one, culminating into some of the dreaded offending organisms. Nearly ninety years after the advent of antibiotics, the issue of sensitivity and resistance to antibiotics has become a matter of concern.

In order to manage the patients with long standing fever, microbiological diagnosis is of utmost importance. Blood cultures are believed to be the "gold standard" for diagnosis in such patients with suspected systemic infection or in particular septicaemia. ${ }^{5-8}$ Upon detection of the organism, the sensitivity profile of the organism is usually investigated. Antibiotic sensitivity and resistance are an important tool to determine the future line of treatment in patients presenting with suspected infection and fever. The current study was conducted to find out the sensitivity and resistance pattern of the organisms isolated, towards a standard set of anti-microbial agents.

\section{METHODS}

\section{Study design and population}

A cross-sectional study was conducted from January, 2018 to March, 2018 in the Department of Microbiology of a tertiary-care hospital in West Bengal. The attendance of the patients to the hospital were random by nature of the process. Since available records and reports were accessible, no further sampling was employed before data collection. The records of the culture and sensitivity examination performed on the blood samples sent from the different wards were studied. The records of the specimens with clinical history of the patients were included in the study. Blood culture samples sent within 24 hours of admission of the adult patients admitted to different wards with history of prolonged fever (fever for $\geq 7$ days) were included in the study excluding the patients who received any antibiotic within last 1 month of admission (based on history provided). If the accompanying clinical information sheet did not contain any information on antibiotic use during in-hospital management, the samples were excluded from the study. Often a common practice is to leave blanks in antibiotic history section of the accompanying clinical information sheet for those patients who did not get any antibiotic. But since this blank section may also indicate unavailable information, therefore they were not considered. If a patient did not receive any antibiotic in that case in samples where it was clearly written, those samples were only included.

\section{Study variables and technique}

The data collection was done using a pre-designed pretested anonymous semi-structured data collection form. This form contained the principal findings of culture and sensitivity examination and also socio-demographic variables available from clinical information sheet. Sociodemographic variables that were available from the clinical information sheet were considered for analysis. The socio-demographic variables included were age, gender and religion of the patients.

The variables pertaining to microbiological examination were considered in two parts. Firstly, appearance of microbial growth was recorded among the studied specimens. Next sensitivity of anti-microbials were tested among the culture positive samples using semiquantitative method.

\section{Data analysis}

Total of 134 reports of the samples were included in the current study. The data were entered in Epi Info 7 software (Centers for Disease Control, Atlanta) and analysed in SPSS (Statistical Package for Social Sciences) version 10.1 software. $^{9,10}$ The descriptive statistics were obtained regarding socio-demographic variables and the patterns (proportions) of sensitivity and resistance towards different antibiotics. The proportion of sensitivity and resistance was calculated among those samples which showed growth, as sensitivity was tested for isolated organisms.

\section{RESULTS}

\section{Background information}

Majority of the samples examined for the current study were from the general medicine wards. Figure 1 shows the distribution of the patients as per their age. Mean age of the patients was 39.33 years $( \pm 12.19$ years $)$. The youngest patient whose blood was examined was aged 19 years while the oldest was 60 years. The distribution of the patients whose blood samples were examined, are depicted in Figure 2 as per gender and religion.

Among the samples examined, $53.73 \%$ belonged to Hindu patients, while $20.20 \%$ belonged to Muslim patients and $5.97 \%$ Christian. The proportion of male and female patients were comparable among the Hindu and Muslims, while all the Christian patients were male. Overall $47.76 \%$ were female patients and remaining were male. Among the Hindu patients majority were male while among Muslims majority were female. 


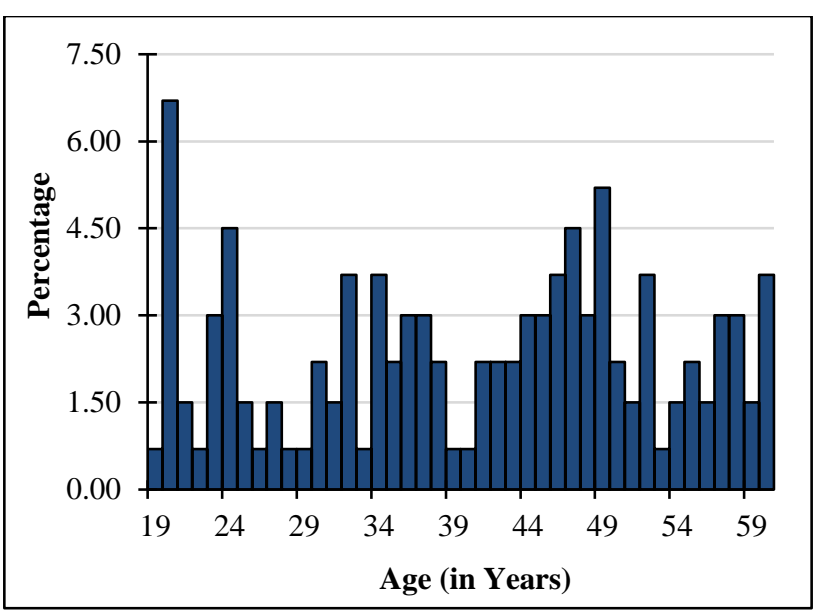

Figure 1: Distribution of the participants as per age $(n=134)$.

\section{Culture isolates}

The description of organisms isolated from the culture are shown in Table 1. Out of the 134 samples $53.73 \%$ did not show any growth on culture. Microbial growth was present in $46.27 \%$ of the samples. Now out of these 62 samples, no sample showed mixed growth i.e. growth of multiple organisms from the same sample. Among the culturepositive samples, majority $(54.84 \%)$ showed growths of Staphylococcus aureus, followed by coagulase negative staphylococcus (16.13\%). In six samples (9.68\%) the organism was identified to be Pseudomonas aeruginosa. Klebsiella, Proteus, Enterococcus, Acinetobacter and Citrobacter was found in $16.15 \%$ of the samples. Candida albicans was isolated from the remaining two samples.

\section{Antibiotic sensitivity and resistance patterns}

Table 2 summarizes the sensitivity and resistance pattern to different anti-microbial agents used. Different cell-wall synthesis inhibitor antibiotics were tested. Among the beta-lactam antibiotics amoxicillin-clavulanic acid was found sensitive in $25.81 \%$ of the samples but was definitively found resistant in $38.71 \%$ cases. In this group the sensitivity of imipenem-cilastatin was observed to be higher (35.48\%). Among the Aminoglycosides sensitivity and resistance to amikacin and netilmycin were comparable ( $41.94 \%$ and $6.45 \%$ respectively). Gentamicin was sensitive in $32.26 \%$ but resistance was observed in $16.13 \%$ of the cases. Among the macrolides azithromycin was tested. A high level of resistance to azithromycin $(35.48 \%)$ was observed in the culture-positive samples, while it was sensitive in $16.13 \%$.

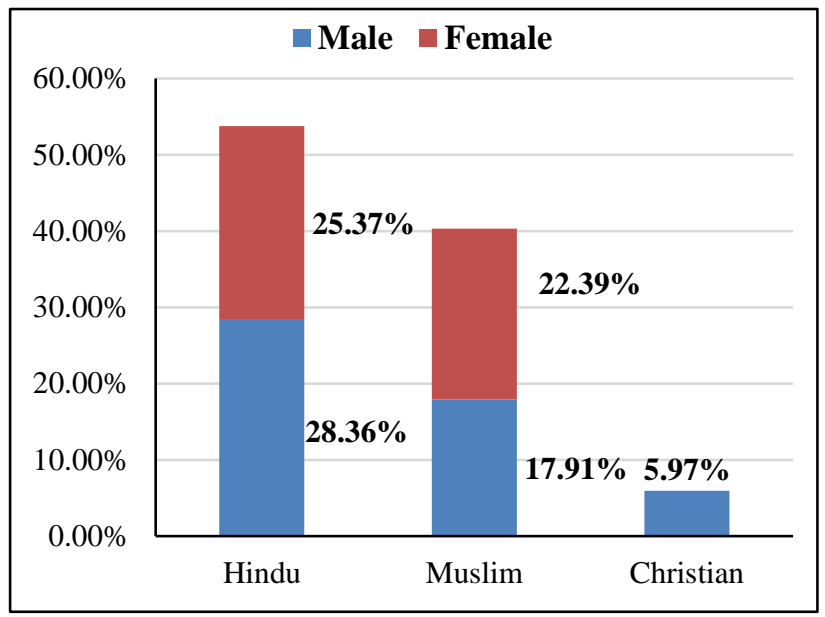

Figure 2: Distribution of the patients as per sex and religion $(n=134)$.

Cefaclor and cefuroxime were the second generation Cephalosporins tested. Cefuroxime was sensitive in $9.68 \%$, while cefaclor was sensitive in two samples. But there was no confirmatory resistance observed to either of these mentioned cephalosporins. Resistance was more pronounced with the third generation cephalosporins. While cefotaxime was resistant in $25.81 \%$, cefoperazone was resistant in $12.90 \%$. Neither of the two was shown to have confirmed sensitivity in any sample.

Table 1: Organisms isolated from the blood culture samples analysed.

\begin{tabular}{|c|c|c|c|}
\hline Variables & Category & Number & Percentage \\
\hline \multirow{2}{*}{$\begin{array}{l}\text { Microbial growth in blood culture sample } \\
(\mathrm{n}=134)\end{array}$} & Growth present & 62 & 46.27 \\
\hline & No growth & 72 & 53.73 \\
\hline \multirow{9}{*}{ Microbial species isolated $(n=62)$} & Acinetobacter $s p .^{*}$ & 2 & 3.23 \\
\hline & Candida albicans & 2 & 3.23 \\
\hline & Citrobacter sp. ${ }^{*}$ & 2 & 3.23 \\
\hline & Coagulase-negative Staphylococcus & 10 & 16.13 \\
\hline & Enterococcus sp. ${ }^{*}$ & 2 & 3.23 \\
\hline & Klebsiella sp. ${ }^{*}$ & 2 & 3.23 \\
\hline & Proteus mirabilis & 2 & 3.23 \\
\hline & Pseudomonas aeruginosa & 6 & 9.68 \\
\hline & Staphylococcus aureus & 34 & 54.84 \\
\hline
\end{tabular}

sp. $=$ species 
Table 2: Sensitivity and resistance pattern towards the anti-microbial agents $(n=62)$.

\begin{tabular}{|c|c|c|c|}
\hline Antibiotic class or group & Anti-microbial agent & Sensitive N (\%) & Resistant N (\%) \\
\hline \multirow{4}{*}{ Beta-lactam } & Amoxycillin-Clavulanic acid & $16(25.81 \%)$ & $24(38.71 \%)$ \\
\hline & Amoxycillin-Sulbactum & $2(3.23 \%)$ & $4(6.45 \%)$ \\
\hline & Piperacillin-Tazobactum & $2(3.23 \%)$ & $2(3.23 \%)$ \\
\hline & Imipenem-Cilastatin & $22(35.48 \%)$ & - \\
\hline \multirow{3}{*}{ Aminoglycosides } & Amikacin & $26(41.94 \%)$ & $4(6.45 \%)$ \\
\hline & Gentamicin & $20(32.26 \%)$ & $10(16.13 \%)$ \\
\hline & Netilmycin & $26(41.94 \%)$ & $4(6.45 \%)$ \\
\hline Macrolides & Azithromycin & $10(16.13 \%)$ & $22(35.48 \%)$ \\
\hline \multirow{2}{*}{$\begin{array}{l}\text { Cephalosporins } \\
2^{\text {nd }} \text { generation }\end{array}$} & Cefaclor & $2(3.23 \%)$ & - \\
\hline & Cefuroxime & $6(9.68 \%)$ & - \\
\hline \multirow{4}{*}{$\begin{array}{l}\text { Cephalosporins } \\
3^{\text {rd }} \text { generation }\end{array}$} & Cefoperazone & - & $8(12.90 \%)$ \\
\hline & Cefotaxime & - & $16(25.81 \%)$ \\
\hline & Ceftriaxone & $14(22.58 \%)$ & $16(25.81 \%)$ \\
\hline & Cefixime & $12(19.35 \%)$ & $34(54.84 \%)$ \\
\hline \multirow{5}{*}{ Fluoroquinolones } & Ciprofloxacin & $6(9.68 \%)$ & - \\
\hline & Moxifloxacin & $8(12.90 \%)$ & $2(3.23 \%)$ \\
\hline & Levofloxacin & $8(12.90 \%)$ & - \\
\hline & Ofloxacin & $10(16.13 \%)$ & $6(9.68 \%)$ \\
\hline & Spurfloxacin & $6(9.68 \%)$ & $2(3.23 \%)$ \\
\hline Glycopeptide & Vancomycin & $30(48.39 \%)$ & - \\
\hline Nitrofurans & Nitrofurantoin & $2(3.23 \%)$ & $4(6.45 \%)$ \\
\hline Folate synthesis inhibitors & Cotrimoxazole & $18(29.04 \%)$ & - \\
\hline \multirow{4}{*}{ Protein synthesis inhibitor } & Doxicycline & $16(25.81 \%)$ & - \\
\hline & Linezolid & $4(6.45 \%)$ & - \\
\hline & Tetracycline & - & $8(12.90 \%)$ \\
\hline & Tigecycline & $2(3.23 \%)$ & $2(3.23 \%)$ \\
\hline \multirow{3}{*}{ Azoles } & Fluconazole & - & $2(3.23 \%)$ \\
\hline & Itraconazole & $2(3.23 \%)$ & - \\
\hline & Ketoconazole & - & $2(3.23 \%)$ \\
\hline
\end{tabular}

The sensitivity and resistance of ceftriaxone was comparable with 14 samples $(22.58 \%)$ being sensitive and $16(25.81 \%)$ resistant. Cefixime however had high level of resistance $(54.84 \%)$, with sensitive in 12 samples $(19.35 \%)$. Among the commonly used fluoroquinolones, ofloxacin was sensitive in $16.13 \%$ cases, but was resistant in $9.68 \%$ cases. Moxifloxacin and levofloxacin both were sensitive in $12.90 \%$ cases. Ciprofloxacin and spurfloxacin was observed to be sensitive in $9.68 \%$ cases. The glycopeptide antibiotic vancomycin showed high level of sensitivity (48.39\%) with no report of confirmed resistance in the examined samples. Cotrimoxazole was the folate synthesis inhibitor tested for sensitivity. It was sensitive in $29.04 \%$ cases with no report of confirmed resistance. Doxycycline was the most sensitive $(25.81 \%)$ protein synthesis inhibitor. Doxycycline and linezolid both did not have any confirmed evidence of resistance. But in case of tetracycline $12.90 \%$ showed evidence of resistance but no confirmed sensitivity was observed. Among the antifungal azoles, fluconazole and ketoconazole showed resistance in both the samples of Candida isolates. In both these samples itraconzole was sensitive.

\section{DISCUSSION}

In the current study the authors identified Staphylococcus aureus to be the most commonly encountered microorganism in the samples of patients with fever for over a week. In their study Karam El-Din et al, observed similar finding. ${ }^{11}$ But in the study by Hossain et al, coagulase negative Staphylococcus were most frequently isolated. ${ }^{12}$ They concluded that the organisms were usually found to be affecting pathogens unless the patient was having some invasive device. In the current study the proportion of coagulase negative Staphylococcus was $16.13 \%$ of the culture-positive samples. In a study by Hall et al, which examined the common contaminants in blood culture, it was observed that Corynebacterium species, Bacillus species other than Bacillus anthracis, Propionibacterium acnes, Micrococcus species, viridans group Streptococci, Enterococci, and Clostridium perfringens were the most common contaminants. ${ }^{13}$ In the current study these microorganisms were not encountered. This may indicate that the level of contamination in the samples examined were less, which implies a high qualitative value of the blood 
culture samples. In the study by Karam El-Din et al, Candida albicans was the most commonly encountered fungal agent. ${ }^{11}$ Candida was the only fungal agent identified in the current study.

In a study conducted by Trojan et al, in Punjab on pus sample isolates of patient it was seen that $S$. aureus showed high susceptibility to vancomycin $(100 \%)$, linezolid $(100 \%)$, imipenem (89\%), and meropenem (84\%) while it showed resistance to ampicillin, amoxicillin-clavulanic acid, ciprofloxacin, and azithromycin. ${ }^{14}$ The current study reported the results of anti-microbial sensitivity from blood-culture samples in a cumulative way. It was observed that overall vancomycin and the aminoglycosides were highly sensitive. Comparable to the study conducted in Punjab, azithromycin, beta-lactam antibiotics and third generation cephalosporins showed evidence of high degree of resistance in the current study.

In the present article evidence regarding anti-fungal residence may not be well-founded due to the lack of fungal isolates. However, the results can definitely indicate the critical level of anti-biotic resistance among the hospital admitted patients. The most widely used antibiotics have shown to become ineffective due to the bacterial resistance. Therefore, it is of utmost importance that constant supervision be imparted on anti-microbial use. The results regarding resistance can help in identifying a trend if analysed cumulatively from different health facilities. Thus, importance of routine culture and sensitivity examination and judicious antibiotic therapy with regular reporting regarding antibiotic resistance cannot be undermined, especially in the current scenario when the 'era of antibiotics' is gradually becoming an 'era of antibiotic resistance'.

\section{ACKNOWLEDGEMENTS}

The authors acknowledge the faculties and staffs associated with Department of Microbiology, Midnapore Medical College and Hospital for their co-operation. The authors also acknowledge the doctors working in the different wards, without their effort the samples with proper clinical information would not have been available for analysis.

\section{Funding: No funding sources}

Conflict of interest: None declared

Ethical approval: The study was approved by the Institutional Ethics Committee Midnapore Medical College \& Hospital, West Bengal

\section{REFERENCES}

1. World Health Organization, editor. Antimicrobial resistance: global report on surveillance. Geneva, Switzerland: World Health Organization; 2014:232.

2. Burchardi H, Schneider H. Economic aspects of severe sepsis: a review of intensive care unit costs, cost of illness and cost effectiveness of therapy. Pharmaco Economics. 2004;22(12):793-813.

3. Tiru B, DiNino EK, Orenstein A, Mailloux PT, Pesaturo A, Gupta A, et al. The Economic and Humanistic Burden of Severe Sepsis. Pharmaco Economics. 2015 Sep;33(9):925-37.

4. Runcie H. Infection in a Pre-Antibiotic Era. J Infect Dis Prev Med. 2015 Jul 31;3(2):1-5.

5. Berendsen EM, Levin E, Braakman R, der Riet-van Oeveren D van, Sedee NJ, Paauw A. Identification of microorganisms grown in blood culture flasks using liquid chromatography-tandem mass spectrometry. Future Microbiol. 2017;12:1135-45.

6. Lamy B, Dargère S, Arendrup MC, Parienti JJ, Tattevin P. How to Optimize the Use of Blood Cultures for the Diagnosis of Bloodstream Infections? A State-of-the Art. Front Microbiol, 2016. Available at:

https://www.ncbi.nlm.nih.gov/pmc/articles/PMC4863 $885 /$.

7. Yamane N. Blood culture: gold standard for definitive diagnosis of bacterial and fungal infections-from the laboratory aspect. Rinsho Byori. 1998 Sep;46(9):88792.

8. Mancini N, Carletti S, Ghidoli N, Cichero P, Burioni $\mathrm{R}$, Clementi M. The Era of Molecular and Other NonCulture-Based Methods in Diagnosis of Sepsis. Clin Microbiol Rev. 2010 Jan;23(1):235-51.

9. Epi InfoTM, CDC. 2017. Available at: https://www.cdc.gov/epiinfo/index.html. Accessed December 2017.

10. IBM SPSS Software, IBM Analytics. Available at: https://www.ibm.com/analytics/spss-statisticssoftware. Accessed August 2018

11. Karam El-Din A-ZA, Mohamed MA, Gad WH, Lotfy GS. Prevalence of microbial pathogens in blood cultures: an etiological and histopathological study. J Taibah Univ Sci. 2010 Jan;3(1):23-32.

12. Hossain B, Islam MS, Rahman A, Marzan M, Rafiqullah I, Connor NE, et al. Understanding Bacterial Isolates in Blood Culture and Approaches Used to Define Bacteria as Contaminants: A Literature Review. Pediatr Infect Dis J. 2016 May;35(5):S45.

13. Hall KK, Lyman JA. Updated Review of Blood Culture Contamination. Clin Microbiol Rev. 2006 Oct;19(4):788-802.

14. Trojan R, Razdan L, Singh N. Antibiotic Susceptibility Patterns of Bacterial Isolates from Pus Samples in a Tertiary Care Hospital of Punjab, India. Int J Microbiol. 2016.

Cite this article as: Ghosh S, Lahiri A, Bera S, Bandyopadhyay S. Anti-microbial sensitivity and resistance of organisms in blood-culture samples from prolonged fever cases: evidence from a tertiary care hospital in West Bengal, India. Int J Basic Clin Pharmacol 2018;7:2173-7. 